\title{
BMJ Open Incidence of post-traumatic epilepsy following paediatric traumatic brain injury: protocol for systematic review and meta-analysis
}

\author{
Frederick P Mariajoseph, ${ }^{1}$ Sarah S Rewell, ${ }^{1,2}$ Terence J O'Brien, ${ }^{1,2,3}$ \\ Bridgette D Semple, ${ }^{1,2,3}$ Ana Antonic-Baker (1) ${ }^{1}$
}

To cite: Mariajoseph FP, Rewell SS, O'Brien TJ, et al. Incidence of post-traumatic epilepsy following paediatric traumatic brain injury: protocol for systematic review and meta-analysis. BMJ Open 2021;11:e054034. doi:10.1136/ bmjopen-2021-054034

- Prepublication history and additional supplemental material for this paper are available online. To view these files, please visit the journal online (http://dx.doi.org/10.1136/ bmjopen-2021-054034).

BDS and AA-B are joint senior authors.

Received 01 June 2021 Accepted 06 0ctober 2021

Check for updates

(c) Author(s) (or their employer(s)) 2021. Re-use permitted under CC BY-NC. No commercial re-use. See rights and permissions. Published by BMJ.

${ }^{1}$ Department of Neuroscience, Monash University, Melbourne, Victoria, Australia

${ }^{2}$ Department of Neurology,

Alfred Health, Prahran, Victoria, Australia

${ }^{3}$ Department of Medicine, The University of Melbourne, Melbourne, Victoria, Australia

Correspondence to

Dr Ana Antonic-Baker;

ana.antonic-baker@monash.edu

\section{ABSTRACT}

Introduction Post-traumatic epilepsy (PTE) is a

recognised complication of traumatic brain injury (TBI), and is associated with higher rates of mortality and morbidity when compared with patients with TBI who do not develop PTE. The majority of the literature on PTE has focused on adult populations, and consequently there is a paucity of information regarding paediatric cohorts. Additionally, there is considerable heterogeneity surrounding the reported incidence of PTE following childhood TBI in the current literature. The primary aim of our study is to summarise reported PTE incidences in paediatric populations to derive an accurate estimate of the global incidence of PTE following childhood TBI. Our secondary aim is to explore risk factors that increase the likelihood of developing PTE. Methods and analysis A systematic literature search of Embase (1947-2021), PubMed (1996-2021) and Web of Science (1900-2021) will be conducted. Publications in English that report the incidence of PTE in populations under 18 years of age will be included. Publications that evaluate fewer than 10 patients, report an alternative cause of epilepsy, or in which a paediatric cohort is not discernable, will be excluded. Independent investigators will identify the relevant publications, and discrepancies will be adjudicated by a third independent investigator. Data extracted will include incidence of PTE, time intervals between TBI and PTE, seizure characteristics, injury characteristics, patient demographics and clinical data. Data extraction will be performed by two independent investigators and cross-checked by a third investigator. A descriptive analysis of PTE incidence will be conducted and a weighted mean will be calculated. If sufficient data are available, stratified meta-analysis of subgroups will also be conducted.

Ethics and dissemination Ethics approval was not required for this study. We intend to publish our findings in a high-quality peer-reviewed journal on completion. PROSPERO registration number CRD42021245802.

\section{INTRODUCTION}

Traumatic brain injury (TBI) refers to an acquired brain injury that occurs subsequent to sudden trauma. The worldwide incidence of TBI is estimated to be 939 cases per 100000 person-years, affecting
Strengths and limitations of this study

- Our protocol was written in accordance with the Preferred Reporting Items for Systematic Reviews and Meta-Analysis Protocols (PRISMA-P) guidelines, and we intend to conduct our study according to the PRISMA guidelines.

- Publishing this protocol ensures that we are transparent with the methods and systems we will be using for this study, to reduce the likelihood of duplication as well as minimise the effects of bias on our study.

- Heterogeneity in reporting of clinical characteristics and outcomes may limit the scope of our analysis.

- Inclusion and exclusion criteria have been defined to cast a wide net, capturing a broad range of published literature regarding the incidence of posttraumatic epilepsy in the paediatric population.

- The exclusion of non-English publications may mean we miss some relevant data.

approximately 69 million individuals annually. ${ }^{1}$ For survivors, TBI is associated with a broad range of physical, cognitive and psychiatric morbidities, including an increased risk of epilepsy. ${ }^{23}$ Post-traumatic epilepsy (PTE) is defined as the occurrence of unprovoked seizures at least 7 days after the initial injury ${ }^{4-6}$ and is a widely acknowledged complication of TBI in both adults and children. ${ }^{78}$ Individuals with a TBI who develop PTE are reported to have a higher mortality rate when compared with patients with a TBI who do not develop PTE. ${ }^{9}$ Furthermore, PTE is associated with poorer chronic outcomes including neurological, intellectual and psychological comorbidities. ${ }^{10-12}$ Given the serious nature of PTE, it is vital to gain a complete understanding of its features and characteristics in order to ultimately improve management and outcomes for patients. Within the current literature, there is no uniform reflection of the incidence of PTE specifically following 
TBI sustained during childhood, as most available data have been concentrated on adult populations, both civilian and military. Making this distinction from adult TBI is important, as developmental age at the time of injury is increasingly recognised as a critical determinant of secondary neuropathology and functional outcomes after TBI. ${ }^{13-15}$ In addition, the causes and mechanisms of TBI may differ in a paediatric population compared with adult cohorts, with a greater proportion attributed to falls and non-accidental injuries. ${ }^{16}$ Against this backdrop, we outline a protocol for conducting a systematic review and meta-analysis to comprehensively investigate the incidence of PTE following paediatric TBI.

The primary outcome measure of this study will be to assess the incidence of epilepsy following childhood (or, paediatric) TBI. Secondary outcome measures will include risk factors such as injury severity, patient demographics and clinical findings and their impact on PTE incidence. In adults, several risk factors for the development of PTE have been identified, including higher injury severity (a higher score on the Glasgow Outcome Scale), skull fracture and the presence of haematomas, ${ }^{17}$ while some studies have identified male sex, interventions and procedures such as mechanical ventilation and acute postinjury seizures, as factors that increase the likelihood of PTE ${ }^{18}{ }^{19}$ Such risk factors have been poorly defined to date in PTE after childhood TBI.

\section{METHODS AND ANALYSIS}

\section{Protocol and registration}

This protocol was written according to the Preferred Reporting Items for Systematic Reviews and Meta-Analyses Protocols guidelines. ${ }^{20}$ The study was designed by discussion with our scientific team with expertise in both TBI and epilepsy research, including translational scientists (SSR, AA-B, BDS) and clinicians (TJO'B) and a clinical trainee (FPM). The protocol is registered in PROSPERO (www.crd.york.ac.uk/prospero/).

\section{Disease of interest}

Between $4 \%$ and $53 \%$ of patients have been reported to experience acute post-traumatic seizures following TBI, defined as provoked seizures occurring within the first 7 days post-injury. ${ }^{72}$ Post-traumatic seizures are a recognised early complication of TBI, which occur as a result of the acute damage sustained by the brain. 'Immediate' seizures are characterised as those occurring within 24 hours of injury), while 'early' seizures refer to those observed within the first week of injury. In contrast, PTE is a distinct phenomenon and refers to 'late'-onset seizures (more than 7 days postinjury), that are recurrent and unprovoked, as a consequence of secondary injury processes that promote hyperexcitable neuronal circuitry. ${ }^{21}$ In line with general consensus in the field, this protocol considers the presentation of late post-traumatic seizures to be adequate for the diagnosis of PTE. ${ }^{22}$

\section{Intervention assessed}

The intervention assessed will be TBI sustained in the context of paediatric or childhood populations.

\section{Control populations}

The control group for our study will be individuals with TBI who do not develop PTE.

\section{Outcome measures}

The primary outcome measure will be the reported incidence of epilepsy. Secondary outcome measures will include the effects of potential factors that may increase the risk of developing PTE, such as patient characteristics as well as clinical and injury characteristics.

\section{Literature search}

A comprehensive literature search will be conducted of three electronic databases: PubMed (1996-2021), Embase (1947-2021) and Web of Science (1900-2021). Key search terms will include variations and synonyms of the following: epilepsy ("epilepsy" or "seizure" or "status epilepticus"), traumatic brain injury ("traumatic brain injury" or "post-traumatic" or "traumatic" or "TBI" or "brain injury"), and pediatric ("paediatric" or "pediatric" or "newborn" or "infan*" or "child*"). For the full list of search terms, please refer to the online supplemental file. Our search strategy will not include any language filters.

\section{Other sources}

To ensure that all relevant articles are included in our study, we will review reference lists of all included studies. Additionally, we will also analyse the reference lists of any previously conducted review articles. The results from our three database searches will be combined with an AND link. All articles yielded from our search will be combined into a single file, and Endnote software (V.X8, Clarivate Analytics) will be used to remove duplicates.

\section{Selection criteria}

\section{Inclusion criteria}

Publications with primary clinical data that report PTE in patients under the age of 18 years at the time of injury will be included.

\section{Exclusion criteria}

Review publications, grey literature, conference abstracts and publications in languages other than English will be excluded from our review. Publications that do not report patient cohorts under the age of 18 years, or from which it is not possible to ascertain paediatric cases, will not be included. Additionally, publications that evaluate fewer than 10 patients will be excluded. Patients with an alternative identifiable cause of epilepsy (eg, epileptogenic lesions unrelated to traumatic insult or an identified pathogenic genomic abnormality) will be excluded.

The web-based systematic review platform Covidence (www.covidence.org; Veritas Health Innovation, Melbourne, Australia) will be used to facilitate the screening process. All studies will undergo abstract and 
title screening by two independent investigators (FPM and SSR), followed by full-text screening by two independent investigators (FPM and SSR). All conflicts during this process will be resolved by a third investigator (BDS, TJO'B or AA-B).

\section{Data collection}

Two independent investigators (FPM and SSR) will extract data from the included studies. In addition to the primary outcome measure, additional variables to be extracted include the following: patient demographics (including age at injury), severity of injury (admission Glasgow Coma Score), diagnostic findings (pathology identified by acute CT), management of TBI (intensive care unit length of stay, intracranial pressure monitoring, interventions such as decompressive craniectomy and administration of anti-seizure medications), the presence/absence of immediate and/or early seizures, mechanism of injury (falls, non-accidental injury, motor vehicle accidents, etc) and the time interval between PTE and TBI (months/years). Pathology identified on CT imaging will be classified according to anatomical location as well as the presence of haemorrhagic contusion, although this classification system is not final and may be revised to better represent the reporting styles of included publication.

\section{Risk of bias assessment}

Risk of bias will be assessed by using a modified version of the Newcastle-Ottawa Scale, a quality assessment scale for cohort studies which encompasses the domains of selection, comparability and outcome. ${ }^{23}$ Each study will be scored and evaluated by two independent investigators (FPM and SSR).

\section{Data analysis}

For each study, we will calculate the proportion of patients with PTE. We will then use Der Simonian and Laird random effect meta-analysis to calculate summary estimate of effect size. The data will be presented as percentage incidence of PTE and its 95\% CIs. Statistical heterogeneity of included studies will be measured with $\mathrm{I}^{2}$. If sufficient data are available, from a minimum of five publications, we will perform stratified meta-analysis and meta-regression to investigate the influence of subgroups on our primary outcome as well as the impact of study design characteristics on results. The effects of subgroups will be determined by using multivariate logistic regression analysis using STATA, with statistical significance set to $\mathrm{p}<0.05$. The subgroups for primary outcome (incidence of PTE following TBI in paediatric population) will be based on additional variables described above in 'Data Collection', for example, patient demographics (age, sex), age of initial TBI, time of follow-up, time of seizure onset and severity of injury. To assess the effects of publication bias, we will perform funnel plot, Egger regression and trim and fill. ${ }^{24}$

\section{Ethics and dissemination}

Ethics approval was not sought or required for this study as data will be obtained from the already published literature. On completion of the systematic review, we will publish the findings in a peer-reviewed academic journal, with raw data available on reasonable request. Additionally, results may also be disseminated in the form of conferences, presentations and seminars.

\section{DISCUSSION}

Our study aims to evaluate and summarise all available published data on the incidence of PTE following paediatric TBI. Seizures in individuals with PTE are often medically intractable, and the promise of new treatments to prevent epileptogenesis after TBI has not yet been realised. ${ }^{25}$ The purpose of our study is to generate improved understanding of PTE in the paediatric population, which will assist clinicians in their approach to the management of both TBI and PTE. Additionally, we hope that this review will identify knowledge gaps and define unanswered research questions, which will provide direction for future research and investigation on this topic, driving towards new strategies to prevent and treat PTE.

To the best of our knowledge, there are currently no other systematic reviews that uniformly consider PTE incidence after childhood TBI. However, our study will complement previous systematic reviews which have investigated the incidence of $\mathrm{TBI}^{26}$ and epilepsy ${ }^{27}$ separately, as well as risk factors of PTE in adult populations. ${ }^{28}$ The specific patient population that we are targeting is the key strength of our review when compared with other studies, as we will obtain an accurate representation of the risk of PTE in a paediatric setting. Focusing exclusively on the paediatric population is important, as it is increasingly recognised that the consequences of TBI are dependent on the developmental age at the time of injury. ${ }^{1429}$ Findings from PTE research in the adult brain may not necessarily apply equally to PTE in the paediatric context, where epileptogenesis after a TBI may be influenced by ongoing brain maturation. By further exploring the risk factors that contribute to PTE in this cohort, this study will also provide valuable information for clinical management.

Our study has a number of potential limitations. First, we are not including non-English publications in our analysis, which may exclude some relevant data. Additionally, we are not setting a limit on the study design of publications, which may affect the quality of studies included. Our analysis may be limited by the heterogeneity in the reporting of patient characteristics and clinical outcomes. Finally, studies that report a mixed population of both adult and paediatric patients will be considered for inclusion, but their contribution to our analysis may be challenging if these two age groups were integrated.

Contributors FPM, BS and AA-B conceived and designed the review. FPM developed the first draft of the protocol manuscript, then SSR, TO'B, BDS and AA-B 
edited the manuscript. FPM, TO'B, BS and AA-B designed the search strategies. All authors read and approved the final manuscript prior to submission for publication.

Funding While this project received no specific funding, the authors are currently supported by funding from the National Health and Medical Research Council of Australia (NHMRC), the US Department of Defense, and Monash University Central Clinical School.

Competing interests None declared.

Patient consent for publication Not applicable.

Provenance and peer review Not commissioned; externally peer reviewed.

Supplemental material This content has been supplied by the author(s). It has not been vetted by BMJ Publishing Group Limited (BMJ) and may not have been peer-reviewed. Any opinions or recommendations discussed are solely those of the author(s) and are not endorsed by BMJ. BMJ disclaims all liability and responsibility arising from any reliance placed on the content. Where the content includes any translated material, BMJ does not warrant the accuracy and reliability of the translations (including but not limited to local regulations, clinical guidelines, terminology, drug names and drug dosages), and is not responsible for any error and/or omissions arising from translation and adaptation or otherwise.

Open access This is an open access article distributed in accordance with the Creative Commons Attribution Non Commercial (CC BY-NC 4.0) license, which permits others to distribute, remix, adapt, build upon this work non-commercially, and license their derivative works on different terms, provided the original work is properly cited, appropriate credit is given, any changes made indicated, and the use is non-commercial. See: http://creativecommons.org/licenses/by-nc/4.0/.

ORCID iD

Ana Antonic-Baker http://orcid.org/0000-0003-4275-7557

\section{REFERENCES}

1 Dewan MC, Rattani A, Gupta S, et al. Estimating the global incidence of traumatic brain injury. J Neurosurg 2018;130:1080-97.

2 Ahmed S, Venigalla $\mathrm{H}$, Mekala HM, et al. Traumatic brain injury and neuropsychiatric complications. Indian J Psychol Med 2017;39:114-21.

3 Bramlett HM, Dietrich WD. Long-Term consequences of traumatic brain injury: current status of potential mechanisms of injury and neurological outcomes. J Neurotrauma 2015;32:1834-48.

4 Fisher RS. An overview of the 2017 ILAE operational classification of seizure types. Epilepsy Behav 2017;70:271-3.

5 Fisher RS, van Emde Boas W, Blume W, et al. Epileptic seizures and epilepsy: definitions proposed by the International League against epilepsy (ILAE) and the International bureau for epilepsy (IBE). Epilepsia 2005;46:470-2.

6 Mendonça GS, Sander JW. Post traumatic epilepsy: there is still much to learn. Arq Neuropsiquiatr 2019;77:373-4.

7 Frey LC. Epidemiology of posttraumatic epilepsy: a critical review. Epilepsia 2003;44:11-17.

8 Statler KD. Pediatric posttraumatic seizures: epidemiology, putative mechanisms of epileptogenesis and promising investigational progress. Dev Neurosci 2006;28:354-63.
9 Lin W-J, Harnod T, Lin C-L, et al. Mortality risk and risk factors in patients with posttraumatic epilepsy: a population-based cohort study. Int J Environ Res Public Health 2019;16:589.

10 Christensen J. The epidemiology of posttraumatic epilepsy. Semin Neurol 2015;35:218-22

11 Englander J, Bushnik T, Duong TT, et al. Analyzing risk factors for late posttraumatic seizures: a prospective, multicenter investigation. Arch Phys Med Rehabil 2003;84:365-73.

12 Mazzini L, Cossa FM, Angelino E, et al. Posttraumatic epilepsy: neuroradiologic and neuropsychological assessment of long-term outcome. Epilepsia 2003;44:569-74.

13 Chong S-L, Lee KP, Lee JH, et al. Pediatric head injury: a pain for the emergency physician? Clin Exp Emerg Med 2015;2:1-8.

14 Giza CC, Mink RB, Madikians A. Pediatric traumatic brain injury: not just little adults. Curr Opin Crit Care 2007;13:143-52.

15 Semple BD, Blomgren K, Gimlin K, et al. Brain development in rodents and humans: identifying benchmarks of maturation and vulnerability to injury across species. Prog Neurobiol 2013;106107:1-16

16 GBD 2016 Traumatic Brain Injury and Spinal Cord Injury Collaborators. Global, regional, and national burden of traumatic brain injury and spinal cord injury, 1990-2016: a systematic analysis for the global burden of disease study 2016. Lancet Neurol 2019;18:56-87.

17 Khalili H, Kashkooli NR, Niakan A, et al. Risk factors for posttraumatic epilepsy. Seizure 2021;89:81-4.

18 Karlander M, Ljungqvist J, Zelano J. Post-Traumatic epilepsy in adults: a nationwide register-based study. J Neurol Neurosurg Psychiatry 2021;92:617-21.

19 Mikkonen ED, Skrifvars MB, Reinikainen M, et al. Posttraumatic epilepsy in intensive care unit-treated pediatric traumatic brain injury patients. Epilepsia 2020;61:693-701.

20 Moher D, Liberati A, Tetzlaff J, et al. Preferred reporting items for systematic reviews and meta-analyses: the PRISMA statement. PLoS Med 2009;6:e1000097.

21 Verellen RM, Cavazos JE. Post-Traumatic epilepsy: an overview. Therapy 2010;7:527-31.

22 Lowenstein DH. Epilepsy after head injury: an overview. Epilepsia 2009;50 Suppl 2:4-9.

23 Wells G, Shea B, O'Connell D. Newcastle-Ottawa quality assessment scale cohort studies. University of Ottawa 2014.

24 Sena ES, van der Worp HB, Bath PMW, et al. Publication bias in reports of animal stroke studies leads to major overstatement of efficacy. PLoS Biol 2010;8:e1000344.

25 Klein P, Friedman A, Hameed MQ, et al. Repurposed molecules for antiepileptogenesis: missing an opportunity to prevent epilepsy? Epilepsia 2020;61:359-86.

26 Nguyen R, Fiest KM, McChesney J, et al. The International incidence of traumatic brain injury: a systematic review and meta-analysis. Can. J. Neurol. Sci. 2016;43:774-85.

27 Fiest KM, Sauro KM, Wiebe S, et al. Prevalence and incidence of epilepsy: a systematic review and meta-analysis of international studies. Neurology 2017;88:296-303.

$28 \mathrm{Xu} \mathrm{T,} \mathrm{Yu} \mathrm{X,} \mathrm{Ou} \mathrm{S,} \mathrm{et} \mathrm{al.} \mathrm{Risk} \mathrm{factors} \mathrm{for} \mathrm{posttraumatic} \mathrm{epilepsy:} \mathrm{a}$ systematic review and meta-analysis. Epilepsy Behav 2017;67:1-6.

29 Pinto PS, Meoded A, Poretti A, et al. The unique features of traumatic brain injury in children. review of the characteristics of the pediatric skull and brain, mechanisms of trauma, patterns of injury, complications, and their imaging findings--part 2. J Neuroimaging 2012;22:e18-41. 